\section{Frequency estimation for L-2- hydroxyglutaric aciduria and hereditary cataract mutations in rescue centre Staffordshire Bull Terriers}

\section{Harvey Carruthers' ${ }^{1}$ Bryan Mclaughlin ${ }^{2}$}

Tay Valley Vet Centre, Perthshire, UK

Animal Health Trust, Suffolk, UK

L-2-hydroxyglutaric aciduria ( 2 2HGA) and hereditary cataract are autosomal recessive disorders found in the SBT; L2HGA is a progressive neurometabolic disorder with significant morbidity and $100 \%$ mortality rate; Hereditary Cataract $(\mathrm{HC})$ is an early onset progressive form of cataract. It is hypothesised that both $\mathrm{HC}$ and L2HGA genetic mutations are present in the UK rescue dog population. The aim of the project was for the first time to estimate the frequency of two disease-associated mutations in SBTs and SBT crosses in Dogs Trust rescue centres.

102 SBT and SBT crosses were selected at random from UK based Dogs Trust rescue centres. Buccal mucosal swabs were obtained from each animal, DNA was extracted and tested for the presence of both the L2HGA and HC mutations by the Animal Health Trust Canine Genetics Department. The genotyping data was used to calculate the mutation frequency.

Six dogs were found to carry a single genetic mutation for L2HGA; five dogs were found to carry a single genetic mutation for HC. No dog was found to be homozygous for either disease, and no dog carried mutations for both diseases. The mutation frequency, for both HC and L2HGA was 0.0012, signifying that approximately $1 \mathrm{dog}$ in 1000 rescue SBT and SBT crosses are likely to be affected by each of the diseases.

This information may prove valuable to rescue centres rehoming large numbers of SBT and their crosses, and may help raise awareness of these debilitating diseases.
"Under the weather?"

Comparing barometric pressure with clinical signs of Chiari malformation and syringomyelia in Cavalier King Charles Spaniels.

\section{William Warman', Edward Ives', Clare Rusbridge $\mathrm{e}^{2,3}$}

\author{
Cambridge University, Cambridge, UK \\ Fitzpatrick Referrals, Godalming, UK \\ Cavalier Matters, Croydon, UK
}

Anecdotally, changes in barometric pressure can lead to changes in degrees of discomfort for dogs affected by Chiari Malformation and Syringomyelia (CM/SM). This prospective, blinded study aimed to determine whether correlation exists between changes in local barometric pressure and the ownerperceived comfort of Cavalier King Charles Spaniels (CKCS) with CM/SM. Such data could allow alteration of medications to reduce episode severity during barometric pressure changes, or the development of a weather warning system to predict exacerbation of clinical signs before this occurs.

Owners of CKCS with CM/SM were requested to complete questionnaires providing background information and daily comfort grading scores for their dogs. Daily comfort grading score and change in comfort score between days were compared to local mean daily barometric pressure and pressure changes both during and between days from Meteorological Office data over a 3 month period. Questionnaires for 22 dogs were analysed, producing 1826 data points. Reported changes from baseline medication were adjusted for in the statistical analysis.

No correlation between mean daily barometric pressure and daily comfort grading score was found (spearman's rho = $0.03, P=0.23$ ). A significant but weak negative correlation between change in mean daily pressure and change in comfort score between days was observed following adjustment for changes in medication $(r=0.05, P=0.026)$. No correlation was found between absolute pressure change and change in comfort score or between absolute pressure change and absolute change in comfort score.

Currently, there is no evidence supporting an association between barometric pressure and the degree of discomfort experienced by CKCS with CM/SM. This appears contrary to the experience of some individuals affected by these conditions. A more objective assessment of a larger population of dogs is required to determine whether or not barometric pressure has an influence on the comfort of some or all CKCS with CM/SM.

A further study by the same authors is in development, aiming to investigate whether there is an association between barometric pressure and comfort scores in people affected by CM/SM. A larger sample size will be sought, allowing more powerful statistical analysis of the results. This canine study has functioned as a useful guide to methods of data collection and analysis that can be used in future canine and human studies on this subject.

This study was funded by Cavalier Matters. 